\title{
Miranda
}

Revue pluridisciplinaire du monde anglophone /

Multidisciplinary peer-reviewed journal on the English-

speaking world

19 | 2019

Rethinking Laughter in Contemporary Anglophone Theatre

\section{Julie Neveux. John Donne. Le Sentiment dans la langue.}

\section{Claire Guéron}

\section{OpenEdition}

\section{Journals}

Édition électronique

URL : http://journals.openedition.org/miranda/21581

DOI : 10.4000/miranda.21581

ISSN : 2108-6559

\section{Éditeur}

Université Toulouse - Jean Jaurès

\section{Édition imprimée}

Date de publication : 7 octobre 2019

\section{Référence électronique}

Claire Guéron, « Julie Neveux. John Donne. Le Sentiment dans la langue. », Miranda [En ligne], 19 | 2019, mis en ligne le 14 octobre 2019, consulté le 16 février 2021. URL : http://journals.openedition.org/ miranda/21581 ; DOI : https://doi.org/10.4000/miranda.21581

Ce document a été généré automatiquement le 16 février 2021.

\section{c) $\$(\Theta)$}

Miranda is licensed under a Creative Commons Attribution-NonCommercial-NoDerivatives 4.0 International License. 


\title{
Julie Neveux. John Donne. Le Sentiment dans la langue.
}

\author{
Claire Guéron
}

\section{RÉFÉRENCE}

Julie Neveux. John Donne. Le Sentiment dans la langue. Paris : éditions Rue d'Ulm, 2013. 208 p. ISBN-10: 2728804974

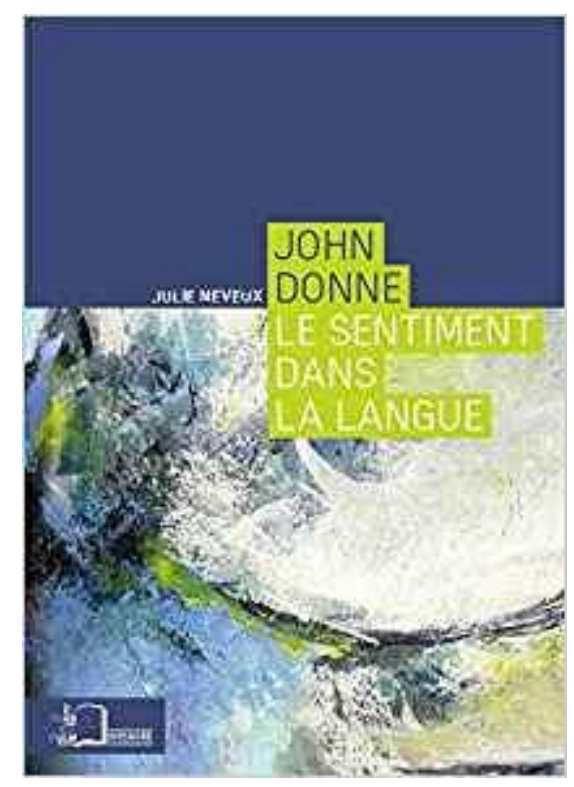

1 Le livre de Julie Neveux aborde l'expression du sentiment dans la poésie et les sermons de John Donne par le biais de la linguistique et de la stylistique. L'auteur défend la thèse selon laquelle la force évocatrice des œuvres de Donne serait attribuable à l'emploi conjugué de la métaphore et de substantifs en -ness ${ }^{1}$, qui donnent un caractère concret à la représentation de l'émotion. Le sentiment, défini comme la perception d'une 
émotion, ne peut être correctement transmis de façon dénotative, car il s'enracine dans une expérience particulière et donc non encore répertoriée dans la «langue propre ", langue du dictionnaire, qui renvoie toujours à un déjà. L'ouvrage de Julie Neveux se donne pour mission de montrer le fonctionnement de la métaphore et des noms en ness dans l'œuvre de Donne, c'est à dire d'analyser l'effet de concret dont sont investies ces deux formes. Dans cette optique, l'auteur associe linguistique énonciative, linguistique cognitive et phénoménologie.

2 Dans un premier chapitre, l'auteur s'attache à définir le concret d'un point de vue cognitif, comme ce qui renvoie à une expérience particulière. Le deuxième chapitre s'appuie sur la philosophie pragmatique de William James pour montrer comment, dans les Dévotions ${ }^{2}$ de Donne, cet effet de concret est obtenu grâce à la nominalisation de l'adjectif par le suffixe -ness, l'effet de singularité passant souvent par le recours à des néologismes comme «beautifulness" ou encore le surprenant « disconsolatedness ». La nominalisation de l'adjectif permet d'ancrer la notion dans une situation particulière et d'évoquer l'émotion incarnée. Le troisième chapitre s'intéresse à la métaphore. L'auteur y démontre que la métaphore est plus concrète que la comparaison, parce qu'elle prend sa source dans l'émotion, plutôt que dans le jugement. La métaphore permet en outre de transmettre le sentiment plus facilement que la comparaison, car la trace de l'énonciateur en est pratiquement effacée, tandis que le « like» de la comparaison implique l'auteur du jugement. Cette idée est illustrée par des exemples tirés de The Bait, The Second Anniversary et Divine Poem 19. Le lyrisme de ces poèmes provient en fait de la fusion de la métaphore et de la comparaison au sein du metaphysical conceit, cette figure de style outrancière que Samuel Johnson, dans The Lives of The Most Eminent English Poets, définissait comme le couplage violent et contrenature de deux notions disparates ${ }^{3}$. L'association de la métaphore et de la comparaison permet de souligner l'insuffisance de la pensée rationnelle, le sentiment se situant "entre métaphore et comparaison, affect et intellect» (116). C'est le principe du «lyrisme indirect » (55) qui restitue le sentiment grâce à l'effet de surprise crée par l'image incongrue.

3 Le quatrième chapitre, "De l'amour à l'humain", applique cette idée de «lyrisme indirect » à l'expression du sentiment amoureux dans Elegy 19, To His Mistress Going to Bed, avant d'envisager la métaphore comme un outil philosophique, permettant une approche phénoménologique de la confusion inhérente à l'expérience humaine. En rendant compte $d u$ "trouble du vivant» (138), la métaphore prend sa place dans l'élaboration d'une langue concrète. Cette langue concrète, libérée du carcan de la dénotation scientifique, passe par une réappropriation du langage à travers un style particulier, dont la métaphore serait la clé de voûte. Le langage métaphorique est en effet le seul à pouvoir formuler l'expérience de façon précise, car il existe une analogie entre la synthèse effectuée par la métaphore et la réciprocité inscrite dans l'expérience phénoménologique du monde.

4 Le cinquième chapitre s'intéresse plus particulièrement à l'expérience de l'amour de Dieu. Il s'agit de montrer comment la métaphore permet de remédier à l'absence de Dieu en inscrivant la divinité dans la chair du croyant, recréant ainsi le mystère de l'incarnation. La métaphore prend alors une dimension mystique. C'est par le biais de la métaphore que les poèmes ou les Dévotions rejoignent le rituel catholique, en dépit de la conversion de Donne au culte anglican. 
Le livre de Julie Neveux apporte un éclairage nouveau sur l'œuvre de John Donne, car il ne s'agit pas d'en réaliser une exégèse mais d'exposer le fonctionnement des formes linguistiques et stylistiques par lesquelles Donne parvient à communiquer l'émotion ressentie à son lecteur, malgré tout ce que l'émotion comporte d'indicible. Cette approche, qui épouse avec originalité le tournant cognitif et phénoménologique pris par les études élisabéthaines et jacobéennes depuis une vingtaine d'années, est convaincante. Les non-spécialistes trouveront peut-être un peu arides les développements théoriques qui structurent l'ouvrage, mais l'auteur propose ici une nouvelle façon de lire Donne qui méritait d'être approfondie. Plus que des interprétations clé en main des œuvres du poète et prédicateur anglais, c'est en effet une méthode qui nous est livrée, et Julie Neveux a pris le temps de détailler le pourquoi et le comment de cette méthode, en convoquant à propos Merleau-Ponty, Spinoza, Paul Ricœur, George Lakoff, Antonio Damasio et William James. Le chapitre 3, pivot de l'ouvrage, est émaillé de plusieurs schémas explicatifs, d'utilité inégale : la plupart du temps, le style rigoureux de l'auteur suffit, et ces illustrations sont plutôt l'occasion d'une pause réflexive.

6 Dans sa préface, Pierre Cotte souligne la difficulté de l'objectif que s'est fixé Julie Neveux, à savoir établir une "phénoménologie du sens » (9) de l'œuvre de Donne. A l'aide d'une démonstration par étapes et d'une attention permanente à la justesse des termes employés, l'auteur remplit son contrat. Le tour de force de Julie Neveux est d'avoir réussi à rendre compte du lyrisme de Donne dans une langue résolument analytique et dénotative.

\section{NOTES}

1. Conçus eux-mêmes comme des « métaphore[s] grammaticale[s)]» (60).

2. Devotions upon Emergent Occasions.

3. «The most heterogeneous ideas are yoked by violence together» (95).

\section{INDEX}

Keywords : John Donne, sentiment/feeling, emotion, cognitive linguistics, metaphor, conceit, concrete language, phenomenology, lyricism

Mots-clés : John Donne, sentiment, émotion, linguistique cognitive, métaphore, conceit, langue concrète, phénoménologie, lyrisme 


\section{AUTEURS}

\section{CLAIRE GUÉRON}

Maître de conférences

Université de Bourgogne Franche-Comté

claire.gueron@u-bourgogne.fr 